\title{
A discrete-time Sliding Window Observer for Markovian Switching System
}

\author{
Abdelfettah Hocine, Mohammed Chadli, Didier Maquin and José Ragot
}

\begin{abstract}
In this paper, a fault detection method is developed for switching dynamic systems. These systems are represented by several linear models, each of them being associated to a particular operating mode. To finding the system operating mode the proposed method is based on mode probabilities and on a new structure of discrete-time observer with a sliding window measurements. This observer results from a combination of a Finite Memory Observer (FMO) and a Luenberger Observer. The stability condition of the observer is formulated in terms of linear matrix inequalities (LMI) using a quadratic Lyapunov function.

The method also uses a priori knowledge information about the mode transition probabilities represented by a Markov chain. The proposed algorithm is of supervised nature where the faults to be detected are a priori indexed and modelled. In this work, the method is applied for the fault detection of a linear system characterized by a model of normal operating mode and several fault models. A comparison with the Generalized PseudoBayesian method shows the validity and some advantages of the suggested method.
\end{abstract}

Index Terms - State estimation, diagnosis, Markovian switching system, multiple model, Lyapunov function, LMI, sliding observer.

\section{INTRODUCTION}

As an evidence, control of systems is becoming more and more sophisticated; that is due to the combined fact that systems are naturally complex but also because it is often desired to manage all things affecting the system. This motivates researches on reliability, availability and security. In this field, FDI (Fault Detection and Isolation) has been developed over the two last decades [8], [4]. A common way to FDI is often based on the state estimation of a process which also produces an estimation of the process output. In general a simple comparison of estimated and measured outputs is used to design a set of residuals that are sensitive to faults. Thus, state estimation is a key point of FDI. Generalized Pseudo-Bayesian first order approach (GPB1) [2] is a powerful tool to track the behaviour evolution of the process; this latter is also based on residual information. Frequently, process can be characterized by one or several models for normal operating conditions and by another set of models describing the different situations of misfunctionning affecting sensors and actuators (that are the consequence of damage of process components). This

Abdelfettah Hocine, Didier Maquin and José Ragot are with Centre de Recherche en Automatique de Nancy, UMR 7039, Nancy-Université, CNRS, 2, Avenue de la Forêt de Haye - 54516 Vandoeuvre les Nancy Cedex, France

Mohammed Chadli is with Centre de Robotique, d'Electrotechnique et d'Automatique Université de Picardie - Jules Verne 7, Rue du Moulin Neuf 80000 Amiens, France set of models can be used to describe the overall behaviour of the process. For this purpose, the multiple model strategy which is often used is exploited in the GPB1 approach. In the topic of state estimation, the GPB have a wide range of applications mainly in tracking targets [1],[2]. In the last few years, the multiple model approach has found a wide spectrum of application, including state estimation [1], [2], [3], [6], control [7] and modeling [5]. In general, a parallel bank of filters is used where each filter is based on a local model representing a particular behaviour of the actual process. The evaluation of the residuals between filter outputs and observed process outputs allows one to design fault detectors. The GPB method is based mainly on the Kalman filter and mode probabilities. In this work, the Kalman filter is replaced by a Sliding Window Observer (SWO).

In the scientific literature, the issue of state estimation of dynamic systems was addressed by different kinds of methods. Among them, those based on the whole process history (i.e. infinite memory) such as classical Luenberger observer can be mentioned. This type of observer is sensitive to modeling errors and it is well known that they sometimes cause a divergence phenomenon as the observer tends to accumulate the errors along the time. Moreover, the infinite memory property inflicts observer insensitivity to recent measurements because it tends to rely more on the process history than current measurements [9]. By construction, it has an inertia (low sensitivity to the recent measurements) which, depending of the application nature, is not always suitable. To overcome this drawback, another class of observers using finite number of delayed input/output measurements on a finite time window was also defined. These observers are denoted using various terms such as finite-memory, receding-horizon or sliding-window estimation [15], [16] and [11]. Finite memory estimation is often accomplished according to the minimization of a least-squares criterion (see among others, [13] and [14]). Unfortunately, finite memory observers are often too sensitive to the output measurement noise [10]. In order to take advantages of both type of observers, this paper proposes a new observer structure combining the Luenberger observer with infinite memory and a Finite Memory observer throughout a simple weighting scheme and introduce this weighted observer in the framework of switching system estimation and diagnosis.

This paper is organized as follows. In section II, the 
development of a finite memory observer is recalled. In section III, a new structure of a sliding window observer is proposed. The asymptotic stability of the proposed observer is studied through a quadratic Lyapunov function. The satisfaction of LMI conditions ([12]) guarantees the boundness of the state estimation error. In section V, we apply the proposed observer to the state estimation of an academic example to illustrate the feasibility of the approach and to compare the results with those obtained using FMO and Luenberger observers. Based on an SWO, section VI presents a method allowing to detect the changing regime within the switching systems framework. Finally, an illustrative example is given to show the validity and some advantages of the suggested method compared with the Generalized Pseudo-Bayesian method.

Notations- Throughout the paper, the following notation is used: $X^{T}$ denotes the transpose of the matrix $X$, $X>0(X \geq 0)$ means that $X$ is symmetric positive definite (semidefinite) matrix and $\lambda_{\min }(X)\left(\lambda_{\max }(X)\right)$ denotes the smallest (largest) eigenvalue of $X$.

\section{A Finite Memory Observer}

A FMO uses the measurements of a finite time interval only. Let us consider the following invariant discrete time system:

$$
\left\{\begin{aligned}
x_{k+1} & =A x_{k}+B u_{k}+G w_{k} \\
y_{k} & =C x_{k}+v_{k}
\end{aligned}\right.
$$

where $x_{k} \in \mathbb{R}^{n}$ is the state vector at time $k, A \in \mathbb{R}^{n \times n}$ is the state matrix, $u_{k} \in \mathbb{R}^{p}$ is the input vector, $B \in \mathbb{R}^{n \times p}$ is the input gain matrix, $G \in \mathbb{R}^{h}$ is the noise gain matrix, $C \in \mathbb{R}^{q \times n}$ is the output gain matrix, $v_{k} \in \mathbb{R}^{q}$ and $w_{k} \in \mathbb{R}^{h}$ are respectively, the state and measurement noises and $y_{k} \in \mathbb{R}^{q}$ is the output of the system.

In the noise-free case, the system is described by:

$$
\left\{\begin{aligned}
x_{k+1} & =A x_{k}+B u_{k} \\
y_{k} & =C x_{k}
\end{aligned}\right.
$$

On the time horizon $[k, k+m-1]$, where the observation window is of size $m$, the output evolution of the system is given by :

$$
\bar{y}_{k}=L_{m} x_{k}+B_{m} \bar{u}_{k}+G_{m} \bar{w}_{k}+\bar{v}_{k}
$$

with the following definitions :

$$
\begin{gathered}
\bar{z}_{k}=\left[\begin{array}{llll}
z_{k}^{T} & z_{k+1}^{T} & \ldots & z_{k+m-1}^{T}
\end{array}\right]^{T}, \quad z \in\{y, u, w, v\} \\
L_{m}=\left[\begin{array}{lllll}
C^{T} & (C A)^{T} & \ldots & \left(C A^{m}\right)^{T}
\end{array}\right]^{T} \\
B_{m}=\left[\begin{array}{ccccc}
0 & 0 & \ldots & \ldots & 0 \\
C B & 0 & \ddots & \ddots & 0 \\
C A B & C B & \ddots & \ddots & \vdots \\
\vdots & \vdots & \ddots & \ddots & 0 \\
C A^{m-2} B & C A^{m-3} B & \ldots & C B & 0
\end{array}\right]
\end{gathered}
$$

$$
G_{m}=\left[\begin{array}{ccccc}
0 & 0 & \ldots & \ldots & 0 \\
C G & 0 & \ddots & \ddots & 0 \\
C A G & C G & \ddots & \ddots & \vdots \\
\vdots & \vdots & \ddots & \ddots & 0 \\
C A^{m-2} G & C A^{m-3} G & \ldots & C G & 0
\end{array}\right]
$$

Let $\tilde{y}_{k}=L_{m} \hat{x}_{k}+B_{m} \bar{u}_{k}$, and the state estimate $\hat{x}_{k-m}$ at the moment $k-m$, can be obtained easily using the least square method, by minimizing the criterion $J_{k}=\left(\tilde{y}_{k}-\bar{y}_{k}\right)^{T}\left(\tilde{y}_{k}-\bar{y}_{k}\right)$ subject to $x_{k}$. We obtain:

$$
\hat{x}_{k}=\alpha \bar{y}_{k}-\beta \bar{u}_{k}
$$

where

$$
\alpha=\left(L_{m}^{T} L_{m}\right)^{-1} L_{m}^{T}, \quad \beta=\left(L_{m}^{T} L_{m}\right)^{-1} L_{m}^{T} B_{m}
$$

The term $\left(L_{m}^{T} L_{m}\right)^{-1}$ exists only if $L_{m}$ is of full rank column. Substituting $\bar{y}_{k}(3)$ in (8) allows to obtain the state estimation error:

$$
e_{k}=\hat{x}_{k}-x_{k}=\alpha \bar{v}_{k}+\gamma \bar{w}_{k}
$$

with $\gamma=\left(L_{m}^{T} L_{m}\right)^{-1} L_{m}^{T} G_{m}$.

From (8) and (10), it is also possible to express the state vector:

$$
x_{k}=\alpha \bar{y}_{k}-\beta \bar{u}_{k}-\alpha \bar{v}_{k}-\gamma \bar{w}_{k}
$$

It can be shown easily that the state estimation error 10 has zero mean and constant variance; the observer is therefore unbiased for centered noises in state and measurements.

The size of the horizon of observation is a parameter that can be adjusted to control the dynamics of the finite memory observer (8). It will be chosen according to system (1) and to the level of measurement noise.

\section{SLIDING WINDOW OBSERVER STRUCTURE}

Considering the equations (1) and (11), the expression of the system state can be also expressed as follows:

$$
\begin{aligned}
x_{k+1} & =\tau\left(\alpha \bar{y}_{k+1}-\beta \bar{u}_{k+1}-\alpha \bar{v}_{k+1}-\gamma \bar{w}_{k+1}\right) \\
& +(1-\tau)\left(A x_{k}+B u_{k}+w_{k}\right)
\end{aligned}
$$

where $\tau$ is a weighting scalar between the instantaneous state and the state computed on the horizon of size $m$.

For this system, the following observer structure is proposed:

$$
\left\{\begin{aligned}
\hat{x}_{k+1} & =\tau\left(\alpha \bar{y}_{k+1}-\beta \bar{u}_{k+1}\right) \\
& +(1-\tau)\left(A \hat{x}_{k}+B u_{k}-K\left(\hat{y}_{k}-y_{k}\right)\right) \\
\hat{y}_{k} & =C \hat{x}_{k}
\end{aligned}\right.
$$

where $K$ is the observer gain to be computed.

To study the performances of this observer structure, we use the dynamics of estimation error $e_{k}=\hat{x}_{k}-x_{k}$ which is given by:

$$
e_{k+1}=\bar{A} e_{k}+(1-\tau) K v_{k}+\tau \alpha \bar{v}_{k+1}+\tau \gamma \bar{w}_{k+1}-(1-\tau) w_{k}
$$

with

$$
\bar{A}=(1-\tau)(A-K C)
$$


The estimation error can be rewritten as follows:

$$
e_{k+1}=\bar{A} e_{k}+F \bar{g}_{k}
$$

where

$$
\bar{g}_{k}=\left[v_{k}, \bar{v}_{k+1}, w_{k}, \bar{w}_{k+1}\right]^{T}
$$

is the vector of noise and

$$
F=[(1-\tau) K, \tau \alpha,(\tau-1) G, \tau \gamma]
$$

is the concatenation of the distribution matrices.

The design of the observer requires the computation of $K$ and $\tau$. For that, the following lemma is needed to proof our main result.

Lemma: let $M$ and $N$ matrices with suitable dimensions. The following property holds [12]:

$$
M^{T} N+N^{T} M \leq M^{T} M+N^{T} N
$$

The proof of the following proposition is based on the analysis of the quadratic Lyapunov function:

$$
V_{k}\left(e_{k}\right)=e_{k}^{T} P e_{k}
$$

and its variation:

$$
\Delta V_{k}\left(e_{k}\right)=e_{k+1}^{T} P e_{k+1}-e_{k}^{T} P e_{k}
$$

Proposition: Suppose that there exists a gain matrix $K$, two positive definite matrices $P>0$ and $Q>0$, and a given positive scalar $\tau$, satisfying the following matrix inequality:

$$
\begin{aligned}
& Q-P+(1-\tau)^{2}(A-K C)^{T} P(A-K C) \\
& +(1-\tau)^{2}(A-K C)^{T} P^{2}(A-K C)<0
\end{aligned}
$$

then the observer (13) has a bounded estimation error, i.e. there exists a positive constant

$$
r^{2}=\|F\|^{2}\left\|\bar{g}_{k}\right\|^{2} \lambda_{\max }(P+I) / \lambda_{\min }(Q)
$$

such that $\Delta V_{k}\left(e_{k}\right)<0$ for $\left\|e_{k}\right\|>r$, where $I$ denotes the identity matrix.

Proof: Let us consider the quadratic Lyapunov function (20), we obtain:

$$
\begin{aligned}
& \quad V_{k+1}\left(e_{k}\right)=e_{k+1}^{T} P e_{k+1} \\
& \quad=e_{k}^{T} \bar{A}^{T} P \bar{A}_{k}+2 e_{k}^{T} \bar{A}^{T} P F \bar{g}_{k}+\bar{g}_{k}^{T} F^{T} P F \bar{g}_{k}
\end{aligned}
$$

the above lemma allows us to write

$$
2 e_{k}^{T} \bar{A}^{T} P F \bar{g}_{k} \leq e_{k}^{T} \bar{A}^{T} P P \bar{A} e_{k}+\bar{g}_{k}^{T} F^{T} F \bar{g}_{k}
$$

substituting (25) in (24), we obtain:

$$
\begin{aligned}
& V_{k+1}\left(e_{k}\right) \leq e_{k}^{T}\left(\bar{A}^{T} P \bar{A}+\bar{A}^{T} P^{2} \bar{A}\right) e_{k}+\bar{g}_{k}^{T} F^{T} F \bar{g}_{k} \\
&+\bar{g}_{k}^{T} F^{T} P F \bar{g}_{k} \\
&=e_{k}^{T}\left(\bar{A}^{T} P \bar{A}+\bar{A}^{T} P^{2} \bar{A}\right) e_{k}+\bar{g}_{k}^{T} F^{T}(P+I) F \bar{g}_{k}
\end{aligned}
$$

Then

$V_{k+1}\left(e_{k}\right) \leq e_{k}^{T}\left(\bar{A}^{T} P \bar{A}+\bar{A}^{T} P^{2} \bar{A}\right) e_{k}+\|F\|^{2}\left\|\bar{g}_{k}\right\|^{2} \lambda_{\max }(P+I)$

Therefore, if there exists $P>0, Q>0$ such that

$\bar{A}^{T} P \bar{A}+\bar{A}^{T} P^{2} \bar{A}-P<-Q$

then

$$
\begin{aligned}
\Delta V_{k}\left(e_{k}\right) & \leq-e_{k+1}^{T} Q e_{k+1}+\|F\|^{2}\left\|\bar{g}_{k}\right\|^{2} \lambda_{\max }(P+I) \\
& \leq-\lambda_{\min }(Q)\left\|e_{k}\right\|^{2}+\|F\|^{2}\left\|\bar{g}_{k}\right\|^{2} \lambda_{\max }(P+I)
\end{aligned}
$$

and $\Delta V_{k}(e(k))<0$ if $\left\|e_{k}\right\| \geq r$. To solve the nonlinear matrix inequality (22), the following sufficient LMI condition in the variables $P>0, Q>0$ and $X$ is proposed:

$$
\left[\begin{array}{ccc}
(1-\tau)^{-2}(P-Q) & (P A-X C)^{T} & (P A-X C)^{T} \\
P A-X C & P & 0 \\
P A-X C & 0 & I
\end{array}\right]>0
$$

The gain observer is given by $K=P^{-1} X$.

It is worthwhile to notice that this observer synthesis guarantees the convergence of the estimation error to a bounded interval defined by (23). This convergence depends on the existence of the matrices $P, Q$ and $K$, that verify the LMI (30) for a given $\tau$.

The choice of the parameter $\tau$ is very important because it directly influences the observer performances. For this reason, we propose in the following section, a method for the determination of the parameter $\tau$.

\section{DISCUSSION}

As described in the previous section the estimation error is bounded by $r$ (23). Obviously the improvement of the performance is based on the minimization of the bound of the estimation error $r$. Therefore, the aim is to minimize the norm of $F$ which depends on parameter $r$ and gain $K$ computed by solving linear matrix inequality (30) which depends directly of $\tau$. Then we are faced with a nonlinear minimization problem.

One possible strategy is to minimize numerically the norm of $F$ with regard to the parameter $\tau$. For this purpose, the matlab function fminbnd has been used. This latter implements a Golden Section Search method [17] coupled with a parabolic interpolation. This optimization procedure finds the minimum of a function of one variable within a fixed interval.

\section{EXAMPLE}

In this section an academic example is introduced to illustrate and to compare the obtained results issued from SWO, FMO and Luenberger observers. This comparison is done using a criterion $E_{N}$ which is the sum, over a given horizon, of the quadratic estimation error.

$$
E_{N}=\sum_{k=1}^{k=N} e_{k}^{T} e_{k}
$$




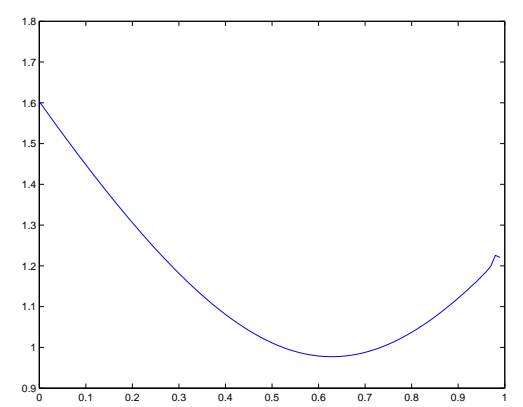

Fig. 1. $\|F\|$ norm evolution according to $\tau$ parameter

Consider the following discrete-time system :

$$
\begin{aligned}
x_{k+1} & =\left[\begin{array}{cc}
0.85 & 0 \\
0 & 0.8
\end{array}\right] x_{k}+\left[\begin{array}{c}
0.18 \\
1.8
\end{array}\right] u_{k}+\left[\begin{array}{l}
0.1 \\
0.1
\end{array}\right] w_{k} \\
y_{k} & =\left[\begin{array}{cc}
1 & 0 \\
0 & 0.5
\end{array}\right] x_{k}+\left[\begin{array}{l}
1 \\
1
\end{array}\right] v_{k}
\end{aligned}
$$

where $v_{k} \in[-0.1 ; 0.1]$ and $w_{k} \in[-0.2 ; 0.2]$ are white Gaussian noises with zero-means.

In order to design the parameter $\tau,\|F\|$ is minimized through the interval $\tau \in[0 ; 1]$, as it is proposed below. This minimization procedure leads (section IV) to the following results.

$$
\begin{gathered}
\tau=0.6285 \\
P=10^{-3}\left[\begin{array}{cc}
50.2 & 49.8 \\
49.8 & 50.1
\end{array}\right] \\
Q=\left[\begin{array}{cc}
50.1 & 49.9 \\
49.9 & 50
\end{array}\right] \\
K=\left[\begin{array}{cc}
0.881 & -0.0722 \\
-0.0282 & 1.7004
\end{array}\right]
\end{gathered}
$$

The evolution of $\|F\|$ is shown in figure 1 , where the window length is $m=7$.

The figures 2 and 3 show the evolution of the estimation errors for the three kinds of observer. It allows us to establish a comparison of the first and the second vector components of the state estimation errors, computed by the different observers. It is worth to remark that the proposed observer provides a good estimation when compared with FMO and Luenberger observers by examining the corresponding error criterion, $\left(E_{N}^{S W O}=5.18\right), \operatorname{OMF}\left(E_{N}^{F M O}=10.39\right)$ and Luenberger $\left(E_{N}^{L B O}=10.22\right)$.

\section{SWO FOR SWITCHING SYSTEMS}

In this section, we consider a system represented by a set of models $M_{i}, i=1, \ldots, r$; each model representing a particular behaviour of the system. The objective is to detect, at each moment, the active model and simultaneously to estimate the state of the system. The transitions from a model to another one are assumed to be described by a Markovian

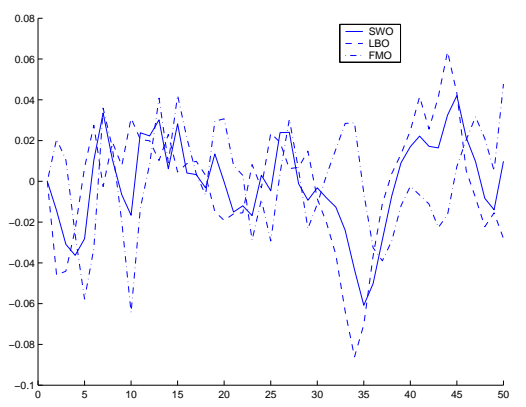

Fig. 2. First state estimation errors

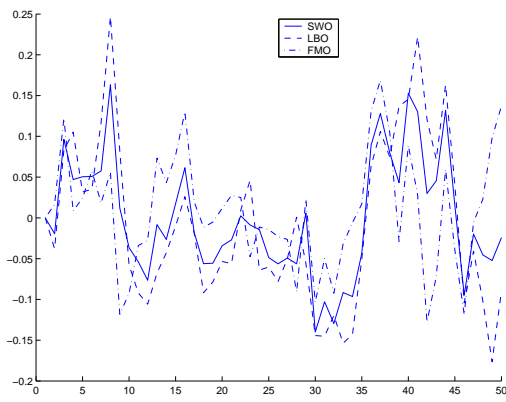

Fig. 3. Second state estimation errors

process governed by an a priori known Markov transition matrix $\Pi$ given by:

$$
\Pi=\left[\begin{array}{ccc}
p_{11} & \cdots & p_{1 r} \\
\vdots & \ddots & \vdots \\
p_{r 1} & \cdots & p_{r r}
\end{array}\right]
$$

where $p_{i j}$ is the mode transition probability from the model $M_{i}$ to the model $M_{j}$; we note $\mu_{j}^{k}$ the probability that the $j^{t h}$ model is active at time $k$.

\section{A. Development of the method}

Consider the $j^{\text {th }}$ model described by:

$$
M_{j}:\left\{\begin{aligned}
x_{k+1} & =A_{j} x_{k}+B_{j} u_{k}+G_{j} w_{k} \\
y_{k} & =C_{j} x_{k}+v_{k}
\end{aligned}\right.
$$

The state estimation of this model is carried out using the SWO described in section III to give the following equations:

$$
\left\{\begin{array}{l}
\hat{x}_{k-m+1}^{j}=\tau_{j}\left(\alpha_{j} \bar{y}_{k-m+1}-\beta_{j} \bar{u}_{k-m+1}\right) \\
+\left(1-\tau_{j}\right)\left(A_{j} \hat{x}_{k-m}+B_{j} u_{k-m}-K_{j}\left(\hat{y}_{k-m}-y_{k-m}\right)\right) \\
\hat{y}_{k-m}=C_{j} \hat{x}_{k-m}^{j}
\end{array}\right.
$$

where the matrices $\alpha_{j}, \beta_{j}$ are built using the definitions (9) after replacing matrices $A, B$ and $C$ by matrices $A_{j}, B_{j}$ and $C_{j}$ related to the $j^{t h}$ model. As before, for the $j^{\text {th }}$ model, $\tau_{j}$ is designed using optimization procedure (section IV) and $K_{j}$ is deduced from (30).

The state estimate at the final time $k$ of the observation window is obtained by integrating the system (2):

$$
\hat{x}_{k}^{j}=A_{j}^{m-1} \hat{x}_{k-m+1}^{j}+T_{j, m} \bar{u}_{k-m+1}
$$




$$
T_{j, m}=\left[\begin{array}{lllll}
\left(A^{m-2} B\right)^{T} & \left(A^{m-3} B\right)^{T} & \ldots & B^{T} & 0
\end{array}\right]^{T}
$$

The state estimate $\hat{x}_{k}$ of the switching system is then computed as a weighted sum of the states of the "local" models:

$$
\hat{x}_{k}=\sum_{j=1}^{r} \hat{x}_{k}^{j} \mu_{k}^{j}
$$

Following the work of Bar-Shalom [2], the probability that model $j$ is in effect at time $k$ is computed in the following way:

$$
\mu_{k}^{j}=P\left\{M_{j}(k) \mid \bar{y}_{k-m+1}\right\}
$$

Define $\tilde{y}_{k-m}$, the observation vector carried out on the horizon $[k-m, k-1]$; we have:

$$
\bar{y}_{k-m+1}=\left[\begin{array}{ll}
\tilde{y}_{k-m}^{T} & y_{k}^{T}
\end{array}\right]
$$

Equation (38) can then be written as:

$$
\mu_{k}^{j}=P\left\{M_{j}(k) \mid \tilde{y}_{k-m}, y_{k}\right\}
$$

Using the Bayes formula, this probability can be transformed into:

$$
\mu_{k}^{j}=\frac{p\left[y_{k} \mid M_{j}(k), \tilde{y}_{k-m}\right] P\left\{M_{j}(k) \mid \tilde{y}_{k-m}\right\}}{\sum_{l=1}^{r} p\left[y_{k} \mid M_{l}(k), \tilde{y}_{k-m}\right] P\left\{M_{l}(k) \mid \tilde{y}_{k-m}\right\}}
$$

In order to alleviate the notations, let us introduce:

$$
L_{i}(k)=p\left[y_{k} \mid M_{i}(k), \tilde{y}_{k-m}\right]
$$

which is a pseudo likelihood function that can be easily computed with the assumption of a normal measurement noise distribution.

Using the total probability theorem, the activation probability of the model $j$ at time $k$, according to the active model at the time $k-1$ can be written as:

$$
\begin{aligned}
& P\left\{M_{j}(k) \mid \tilde{y}_{k-m}\right\}= \\
& \quad \sum_{i=1}^{r} P\left\{M_{j}(k) \mid M_{i}(k-1), \tilde{y}_{k-m}\right\} P\left\{M_{i}(k-1) \mid \tilde{y}_{k-m}\right\}
\end{aligned}
$$

To obtain a recurrence on the computation of the $\mu_{k}^{j}$, we define the following approximation:

$$
P\left\{M_{i}(k-1) \mid \tilde{y}_{k-m}\right\} \approx P\left\{M_{i}(k-1) \mid \bar{y}_{k-m}\right\}=\mu_{k-1}^{i}
$$

That means that the information given by the observation vector $y_{k-m}$ defined on the horizon $[k-m, k-1]$ is not very important and can be neglected (which depends obviously on the selected horizon). In this case, considering equations (41) to (44) and noticing that, by definition, $P\left\{M_{j}(k) \mid M_{i}(k-1), \tilde{y}_{k-m}\right\}=p_{i j}$, the following recurrence on the probability that the system operates according to the model $j$ at the moment $k$ can be established:

$$
\mu_{k}^{j}=\frac{L_{j}(k) \sum_{i=1}^{r} p_{i j} \mu_{k-1}^{j}}{\sum_{l=1}^{r} L_{l}(k) \sum_{i=1}^{r} p_{i l} \mu_{k-1}^{j}}
$$

\section{B. Fault models}

An actuator fault can be modelled by "modifying" an appropriate column of the control input matrix $B$. Thus, a fault on the $i^{t h}$ actuator is described by writing the following equation:

$$
x_{k+1}=A x_{k}+\left(B+\Delta B_{i}\right) u_{k}+w_{k}
$$

where $\Delta B_{i}$ is a matrix with the same dimension of $B$; all of its columns are null except the $i^{\text {th }}$ which characterizes the fault on the $i^{\text {th }}$ actuator.

On a same way, a sensor fault is described by:

$$
y_{k}=\left(C+\Delta C_{i}\right) x_{k}+v_{k}
$$

where $\Delta C_{i}$ is a matrix with the same dimension of $C$; all of its columns are null except the $i^{\text {th }}$ which characterizes the fault on the $i^{t h}$ sensor.

\section{An application example}

For the application of the suggested method, we consider a model of normal operating $\left(A_{1}, B_{1}, C_{1}\right)$, a model of actuator fault $\left(A_{2}, B_{2}, C_{2}\right)$ and a model of sensor faults $\left(A_{3}, B_{3}, C_{3}\right)$, with the various matrices defined by:

$$
\begin{gathered}
A_{i}=\left[\begin{array}{cc}
0.45 & 0 \\
0 & 0.4
\end{array}\right], i=1 \ldots 3 \\
B_{1}=\left[\begin{array}{ll}
0.1815 & 1.7902
\end{array}\right]^{T}, C_{1}=\left[\begin{array}{ll}
1 & 0 \\
0 & 1
\end{array}\right], \\
B_{2}=\left[\begin{array}{ll}
1.1815 & 1.7902
\end{array}\right]^{T}, C_{2}=\left[\begin{array}{cc}
1 & 0 \\
0 & 1
\end{array}\right], \\
B_{3}=\left[\begin{array}{ll}
0.1815 & 1.7902
\end{array}\right]^{T}, C_{3}=\left[\begin{array}{cc}
1.5 & 0 \\
0 & 1.5
\end{array}\right] .
\end{gathered}
$$

Using the optimization procedure described in section 4 for each operating model, we obtain

$$
\begin{gathered}
\tau_{1}=0.2025, \tau_{2}=0.2025, \tau_{3}=0.3025 . \\
K_{1}=\left[\begin{array}{cc}
0.5337 & -0.0340 \\
-0.0840 & 0.4249
\end{array}\right] . \\
K_{2}=\left[\begin{array}{cc}
0.5337 & -0.0340 \\
-0.0840 & 0.4249
\end{array}\right] \\
K_{3}=\left[\begin{array}{cc}
1.3406 & 0.8184 \\
-1.1535 & -0.6226
\end{array}\right]
\end{gathered}
$$

To test the method, the following scenario was established: initially the system normally operates, then at time 100 , an actuator fault occurs, at time 500, the system returns to the normal operating mode and, at time 800 , sensor faults are introduced.

The results are presented at the figures 4,5 and 6 where the changes of mode clearly appear; the mode probabilities of the corresponding models, in their respective operation domains, fluctuate around one and thus a detection of the fault is carried out. Clearly, the results of the suggested method are better than those of GPB1 method. This fact can 


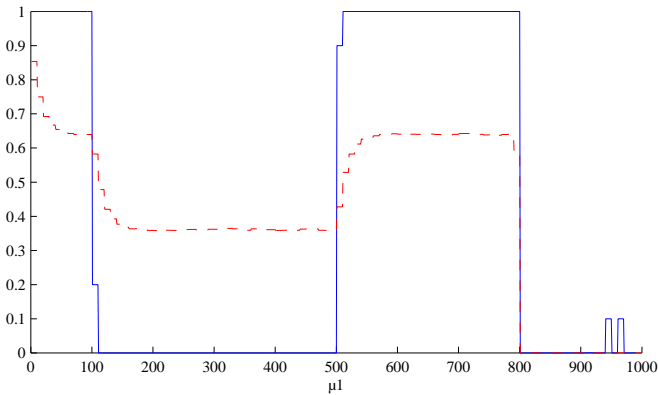

Fig. 4. Activation probability of model 1

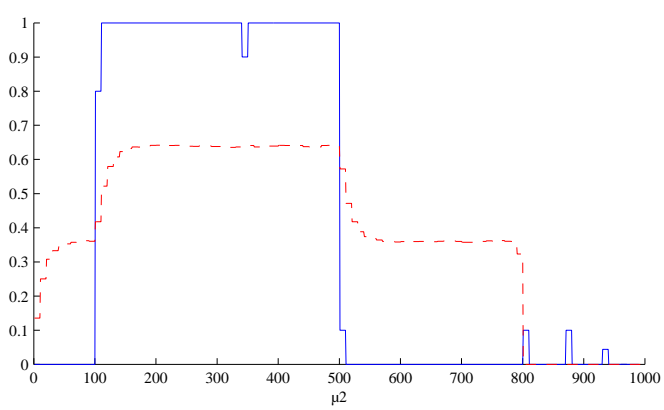

Fig. 5. Activation probability of model 2

be explained by the sensitivity of GPB1 to the noise affecting the system state and measurement (the signal to noise ratio in the example is equal to $10 \%$ ). This allows us to conclude that the use of SWO, for fault detection in switching systems, is less sensitive to noise than traditional GPB methods.

\section{CONCLUSION}

In this work, a structure of a Sliding Window observer (SWO) is proposed to handle the FDI issue. The SWO is based on a combination of the Finite Memory Observer (FMO) and the Luenberger Observer. In the first part, the synthesis conditions of the proposed SWO is addressed under LMI formulation using a quadratic Lyapunov function. The obtained conditions guarantee the convergence of the SWO for a given interval of state estimation error. The weighting parameter $\tau$ is obtained from optimization procedure based on the Golden Section Search minimization

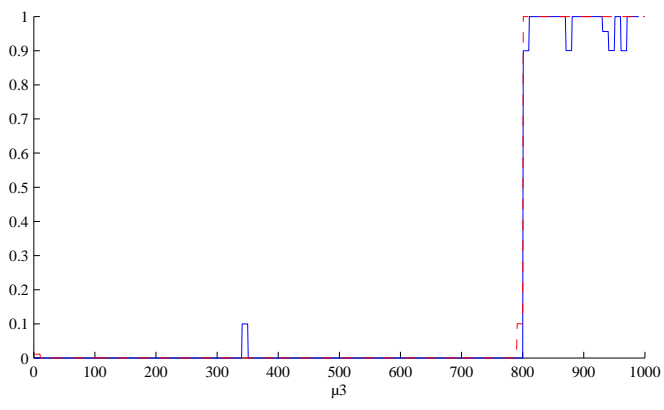

Fig. 6. Activation probability of model 3 method. In the second part, the proposed observer is applied within the framework of Markovian switching systems for which the switching event between models must be detected. Comparison of the obtained results with those of GPB approach was carried out on a computer simulation example. The use of the proposed SWO, based on two kinds of observer methods, in contrast to the GPB method which uses Kalman filter, gives better results, especially in the presence of noises on state system and modeling errors.

\section{REFERENCES}

[1] Y. Bar-Shalom and K.C. Chang and H.A. Blom, "Tracking a manoeuvering target using input estimation versus interacting multiple model algorithm", IEEE Transactions on Aerospace and Electronic Systems,vol. 25, pp. 296-300, 1989.

[2] Y. Bar-Shalom, Multitarget-multisensor tracking: advanced applications. Artech House, 1990.

[3] Y. Bar-Shalom and X. Li, Estimation and tracking: principles, technique and software. Artech House, 1993.

[4] M. Blanke and M. Kinnaert and J. Lunze and M. Staroswiecki, Diagnosis and fault-tolerant control. Springer, 2003.

[5] K. Gasso and G. Mourot and J. Ragot, "Structure identification in multiple model representation: elimination and merging of local models", 40th Conference on Decision and Control, Orlando, 2001.

[6] ZP.D. Hanlon and P.S. Maybeck, "Interrelationship of single-filter and multiple-Model adaptative algorithms", IEEE Transactions on Aerospace and Electronic Systems,vol. 34, pp. 934-946, 1998.

[7] R. Murray-Smith and T.A. Johansen, Multiple Model Approaches to Modelling and Control. Taylor and Francis, 1997.

[8] R. Patton, P.M. Frank and R. Clark, Fault diagnosis in dynamic systems. Prentice Hall, 1989.

[9] Y. Zhang and J. Jiang, "State estimation and fault detection by a bank of continuous finite-memory filters", IFAC World Congress, San Francisco, USA, vol. D, pp. 177-182, 1996.

[10] A. Hocine, D. Maquin and J. Ragot, "Finite memory observer for switching systems: Application to diagnosis", 16th IFAC World Congress, Prague, Czech Republic, 2005.

[11] A. Alessandri, "Design of sliding-mode observers and filters for nonlinear dynamic systems", 39th IEEE Conference on Decision and Control, Sydney, Australia, pp. 2593-2598, 2000.

[12] S. Boyd, L. El Ghaoui, E. Feron and V. Balakrishnan, Linear Matrix Inequalites in System and control Theory. Philadelphia: SIAM, 1994.

[13] G. J. Bierman, "Fixed-memory least squares filtring", IEEE Transactions on Information Theory, vol. IT-21,pp. 690-692, Nov. 1975.

[14] K. V. Ling and K. W. Lim, "Receding horizon recursive state estimation", IEEE Transactions on Automatic Control, vol. AC-44, pp. 1750-1753, Sept. 1999.

[15] W. Nuninger, F. Kratz, J. Ragot, "Finite memory generalised state observer approach for failure detection in dynamic systems", 37th IEEE Conference on Decision and Control, Tampa, Florida USA, pp. 581-585, 1998.

[16] W.H. Kwon, P.S. Kim and "A receding horizon unbiased FIR filter for discret-time state space models". Automatica, 38, pp. 545-551, 2002.

[17] W. Cheney and D. Kincaid, Numerical Mathematics and Computing. Third Edition, Brooks/Cole Publishing Company, 1994. 